\title{
Correction to: Probability of default estimation in credit risk using a nonparametric approach
}

\author{
Rebeca Peláez Suárez ${ }^{1}$ (D) Ricardo Cao Abad ${ }^{2}$. Juan M. Vilar Fernández ${ }^{2}$ \\ Published online: 18 December 2020 \\ ๑) Sociedad de Estadística e Investigación Operativa 2020
}

\section{Correction to: TEST}

\section{https://doi.org/10.1007/s11749-020-00723-1}

The authors would like to correct the errors in the publication of the original article. The correction details are given below:

In page 7, the superscript ' 2 ' that appears in the denominator of the asymptotic expression of Bias $\widehat{P D}(t \mid x))$ has to be removed. The correct expression is as follows:

$$
\operatorname{Bias}(\widehat{P D}(t \mid x))=\frac{(1-P D(t \mid x)) B_{0}(t \mid x)-B_{0}(t+b \mid x)}{S(t \mid x)} h^{2}+o\left(h^{2}\right)+O\left(\frac{1}{n h}\right)
$$

The superscript ' 2 ' that appears in the denominator of the expression of $\alpha_{1}$ in Equation (15) has to be removed. The correct expression is as follows:

$$
\alpha_{1}=\frac{(1-P D(t \mid x)) B_{0}(t \mid x)-B_{0}(t+b \mid x)}{S(t \mid x)} h^{2}+o\left(h^{2}\right)
$$

Publisher's Note Springer Nature remains neutral with regard to jurisdictional claims in published maps and institutional affiliations.

The original article can be found online at https://doi.org/10.1007/s11749-020-00723-1.

$凶$ Rebeca Peláez Suárez

rebeca.pelaez@udc.es

Ricardo Cao Abad

ricardo.cao@udc.es

Juan M. Vilar Fernández

juan.vilar@udc.es

1 Research Group MODES, Department of Mathematics, CITIC, University of A Coruña, A Coruña, Spain

2 Research Group MODES, Department of Mathematics, CITIC, University of A Coruña, ITMATI, A Coruña, Spain 